\title{
Selective implantation of durable left ventricular assist devices as primary therapy for refractory cardiogenic shock
}

\author{
Amit Pawale, MD, ${ }^{a}$ Yosef Schwartz, BS, ${ }^{\mathrm{a}}$ Shinobu Itagaki, MD, ${ }^{\mathrm{a}}$ Sean Pinney, MD, ${ }^{\mathrm{b}}$ David H. Adams, MD, ${ }^{\mathrm{a}}$ \\ and Anelechi C. Anyanwu, MD
}

\section{ABSTRACT}

Objective: Surgical therapy for refractory primary cardiogenic shock is largely based on emergent placement of extracorporeal membrane oxygenation or short-term ventricular assist devices. We have adopted a strategy of routine implantation of durable left ventricular assist devices (LVAD) as initial therapy for refractory cardiogenic shock, in patients who are potential candidates for heart transplantation, and report our experience.

Methods: Retrospective review of 43 consecutive patients with refractory shock caused by acute myocardial infarction $(n=21)$ or acute decompensated heart failure $(n=22)$ who were treated with primary implantation of a durable LVAD in a single institution.

Results: All patients received durable LVAD (axial flow, $\mathrm{n}=37$; centrifugal, $\mathrm{n}=4$; pulsatile, $\mathrm{n}=2$ ), with concurrent placement of right ventricular assist device (RVAD) in 5 patients $(12 \%)$. One patient had delayed RVAD implantation. Mean operative time was 362 minutes and mean cardiopulmonary bypass time was 94 minutes. Twenty patients underwent concurrent cardiac procedures. Major early adverse events included operative mortality $14 \%(6 / 43)$, reoperation for bleeding 7\% (3/43), and stroke 4.7\% (2/43). Median time on mechanical ventilation was 3.5 days, ICU stay 9 days, and hospital stay 25 days. Kaplan-Meier survival was $82.7 \pm 6.0 \%$ at 6 months and $73.9 \pm 8.0 \%$ at 12 months. Using competing analysis, the cumulative incidence of transplantation was $10.3 \pm 5.0 \%$ at 6 months and $30.8 \pm 7.9 \%$ at 1 year.

Conclusions: Our data challenge the notion that patients in refractory cardiogenic shock are best served by an initial period of stabilization with temporary devices. Primary implantation of durable LVADs in cardiogenic shock can yield good midterm outcomes and may have potential benefits. (J Thorac Cardiovasc Surg 2018;155:1059-68)

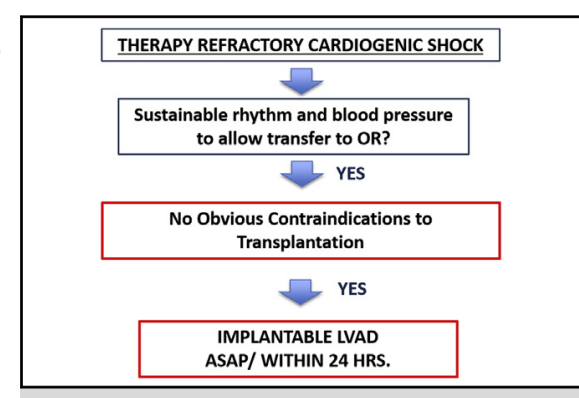

Mount Sinai Algorithm for Management of Refractory Cardiogenic Shock.

\section{Central Message}

Implantation of durable LVADs as primary therapy in refractory cardiogenic shock was feasible and is associated with good outcomes in a single center.

\section{Perspective}

The recommended mechanical support therapy for patients in refractory shock with multiorgan failure is extracorporeal membrane oxygenation. In this study, we present data on an alternative strategy of direct implantation of a durable left ventricular assist device. With this approach, patients may experience shorter periods of hospitalization, fewer reinterventions, and, possibly, better outcomes.

See Editorial Commentary page 1069

See Editorial page 1056.
Traditionally, the mainstay of therapy for refractory cardiogenic shock caused by acute myocardial infarction (AMI)

\footnotetext{
From the ${ }^{\mathrm{a} C a r d i o v a s c u l a r}$ Surgery and ${ }^{\mathrm{b}}$ Cardiovascular Institute, Mount Sinai Hospital, New York, NY.

Read at the 96th Annual Meeting of The American Association for Thoracic Surgery, Baltimore, Maryland, May 14-18, 2016.

Received for publication June 15, 2016; revisions received July 17, 2017; accepted for publication Oct 1, 2017; available ahead of print Dec 20, 2017

Address for reprints: Anelechi C. Anyanwu, MD, Department of Cardiothoracic Surgery, Mount Sinai Medical Center, 1190 Fifth Ave, New York, NY 10029 (E-mail: anelechi.anyanwu@mountsinai.org). 0022-5223/\$36.00

Copyright (C) 2017 Published by Elsevier Inc. on behalf of The American Association for Thoracic Surgery

https://doi.org/10.1016/j.jtcvs.2017.10.136
}

and acute decompensated heart failure (ADHF) has been extracorporeal membrane oxygenation (ECMO) support and temporary percutaneous left ventricular assist device (LVAD) support as a first step. ${ }^{1}$ The 2013 International Society for Heart and Lung Transplantation guidelines for

Scanning this $\mathrm{QR}$ code will take you to a supplemental video. To view the AATS Annual Meeting Webcast, see the URL next to the webcast thumbnail.

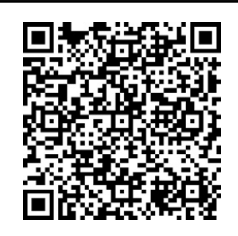




$\begin{array}{ll}\text { Abbreviations } \\ \text { ADHF } & =\text { Acute decompensated heart failure } \\ \text { AMI } & =\text { Acute myocardial infarction } \\ \text { ECMO } & \text { Extracorporeal membrane } \\ & \text { oxygenation } \\ = & \text { Intensive care unit } \\ \text { ICU } & \text { Mechanical Assisted Circulatory } \\ \text { INTERMACS } & \text { Interagency Registry for } \\ & \text { Support } \\ \text { LVAD } & =\text { Left ventricular assist device } \\ \text { RVAD } & =\text { Right ventricular assist device }\end{array}$

mechanical circulatory support recommend short-term mechanical devices for management of acutely decompensated patients with heart failure failing maximal medical therapy (class I, level of evidence C). The guidelines also recommend use of temporary mechanical support in patients with multiorgan failure, sepsis, or on mechanical ventilation to allow successful optimization of clinical status and neurologic assessment before placement of a long-term mechanical circulatory support device (class I, level of evidence C). ${ }^{2}$ The rationale for this recommendation is that patients in cardiogenic shock are too sick to tolerate surgery for implantation of durable LVAD; are more likely to bleed as a result of coagulopathy; are more prone to surgical complications; are more likely to require right ventricular assist devices (RVADs); will frequently not survive the shortterm, regardless of institution of circulatory support (hence resulting in unnecessary incremental cost and resource use if a durable LVAD was placed); and would be more likely to survive implantation of a durable LVAD after a period of optimization with short-term support.

We have previously reported ${ }^{3}$ that primary use of durable LVADs may yield acceptable clinical outcomes in refractory cardiogenic shock and may reduce cost and resource use by avoiding the prolonged intensive care hospitalizations, morbidity, and repeat interventions typically associated with temporary support devices. We report an updated experience of our approach of using durable LVADs as primary therapy for patients with refractory cardiogenic shock.

\section{METHODS \\ Study Design}

Between 2007 and 2016, 315 patients received implantable LVADs in our center, 43 of which were placed as primary therapy for refractory cardiogenic shock caused by AMI or ADHF. This study is a retrospective study of these 43 patients who underwent LVAD implantation as primary therapy for cardiogenic shock in our center. Clinical data were extracted from our departmental cardiac surgery reporting databases. These registries are approved for use in research by the institutional review board, with patient consent waived. We defined therapy-refractory cardiogenic shock as cardiogenic shock with indication of worsening end-organ hypoperfusion (progressive hepatorenal dysfunction, declining mental status, and respiratory failure) or progressive tissue hypoxia (increasing blood lactate level and low venous oxygen
TABLE 1. Patient demographics

\begin{tabular}{lc}
\hline Age (y) & $54.9 \pm 10.8$ \\
Males & $38(88)$ \\
\hline Acute MI & $21(49)$ \\
\hline Decompensated heart failure & $22(51)$ \\
CPR within 24 h before operation & $8(18.6)$ \\
\hline Unknown neurologic status & $5(11.6)$ \\
\hline Preoperative mechanical ventilation & $25(58.1)$ \\
\hline IABP & $32(74.4)$ \\
\hline Percutaneous LVAD & $7(16.3)$ \\
\hline Potent antiplatelet therapy within $48 \mathrm{~h}$ & $16(40)$ \\
\hline Previous sternotomy & $5(11.6)$ \\
Creatinine (mg/dL) & $2.0 \pm 1.3$ \\
\hline Bilirubin (mg/dL) & $2.7 \pm 3.4$ \\
\hline Aspartate aminotransferase (U/L) & $934 \pm 2896$ \\
\hline Lactate (mmol/L) & $3.1 \pm 3.0$ \\
\hline Pulmonary capillary wedge pressure (mm Hg) & $28.2 \pm 6.8$ \\
\hline Right atrial pressure (mm Hg) & $19.4 \pm 9.9$ \\
\hline Pulmonary artery systolic pressure (mm Hg) & $49.7 \pm 11.2$ \\
\hline $\begin{array}{l}\text { Values are number }(\%) \text { or mean } \pm \text { standard deviation unless otherwise indicated. } M I, \\
\text { Myocardial infarction; } C P R, \text { cardiopulmonary resuscitation; } I A B P, \text { intra-aortic } \\
\text { balloon pump; } L V A D \text {, left ventricular assist device. }\end{array}$ \\
\hline
\end{tabular}

saturation), concomitant with sustained hypotension, despite adequate intravascular volume and high doses of inotropes and vasopressors. Patients in cardiogenic shock requiring repeated drug boluses to maintain arterial blood pressure, patients with unstable ventricular arrhythmias, and patients requiring cardiopulmonary resuscitation were also regarded as therapy refractory. Data were abstracted from institutional databases on demographic, process, and outcome variables. The primary end point was all-cause mortality. Operative mortality was defined as any death within 30 days or at any time during the same hospital stay. Stroke was defined as a temporary or permanent new neurologic deficit with computed tomographic evidence of brain infarction or hemorrhage. Limb complications were defined as any limb ischemic complication needing surgical intervention. Potent antiplatelet drugs were defined as any of high-dose clopidogrel, prasugrel, glycoprotein $\mathrm{IIb} / \mathrm{III}$ a inhibitors, or ticagrelor.

\section{Patients}

Patient demographics are described in Table 1. Patients ranged from 33 to 78 years of age and were predominantly male $(n=38,88 \%)$. Twentytwo patients had AMI (51\%) and 21 had ADHF (49\%). The 2 groups were similar in demographics other than higher bilirubin and lower platelet count in the ADHF compared with AMI group $(4.5 \mathrm{mg}$ vs $1 \mathrm{mg} / \mathrm{dL}$; $P=.0003$ and 129,000 vs $194,000 / \mu \mathrm{L} ; P=.018$ respectively). Hemodynamics were notable for a lower mean cardiac index $\left(1.61 / \mathrm{min} / \mathrm{m}^{2}\right.$ vs $2.1 \mathrm{l} / \mathrm{min} / \mathrm{m}^{2}$ ) and higher systolic pulmonary artery pressure (55 vs $44 \mathrm{~mm} \mathrm{Hg}$ ) in the patients with ADHF compared with AMI respectively. Mean right atrial pressure ( $21 \mathrm{vs} 18 \mathrm{~mm} \mathrm{Hg} ; P=.5$ ) and pulmonary artery wedge pressure ( 30 vs $27 \mathrm{~mm} \mathrm{Hg} ; P=.3$ ) were similar in both groups. In patients with post-AMI cardiogenic shock, the median duration from myocardial infarction to LVAD implantation was 3 days (range, 1-23 days). Seven patients had a preexisting percutaneous LVAD placed before referral to the LVAD team but yet remained in cardiogenic shock. Five had a limited flow percutaneous LVAD (Impella 2.5 heart pump [Abiomed Inc, Danvers, Mass]), whereas 2 patients had a TandemHeart percutaneous LVAD (CardiacAssist, Inc, Pittsburgh, Pa). 

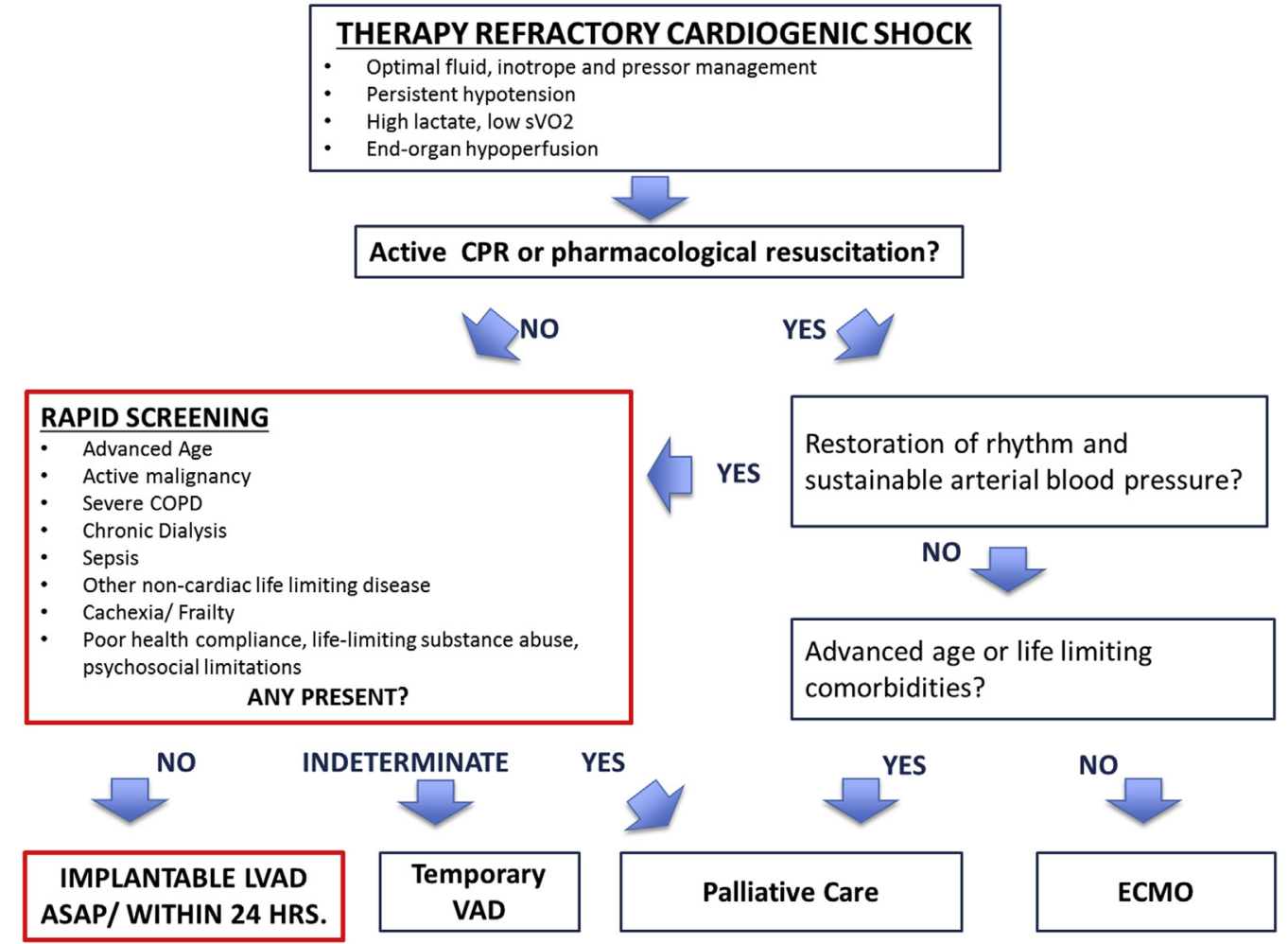

FIGURE 1. Mount Sinai Medical Center algorithm for management of refractory cardiogenic shock. $C P R$, Cardiopulmonary resuscitation; $C O P D$, chronic obstructive airway disease; $L V A D$, left ventricular assist device; $A S A P$, as soon as possible; $V A D$, ventricular assist device; $E C M O$, extracorporeal membrane oxygenation.

\section{Treatment Strategy}

Our strategy for stratification and management of patients in refractory cardiogenic shock is summarized in Figure 1. Typically, patients in refractory cardiogenic shock (1) were on high-dose inotropes, with or without an intra-aortic balloon pump; (2) had a preexisting percutaneous LVAD, often placed during diagnostic or therapeutic cardiac catheterization, but yet still remained with poor tissue perfusion; (3) had malignant ventricular arrhythmias that resulted in poor perfusion; or (4) were undergoing cardiopulmonary resuscitation after cardiac arrest.

After the patients were referred to the LVAD team, a rapid evaluation was performed with the goal to ascertain presence of (1) factors that make short or long-term survival after LVAD implantation unlikely, (2) obvious contraindications to heart transplantation, and (3) psychosocial, logistic, or financial barriers to successful long-term LVAD support. Patients are typically evaluated by a heart failure cardiologist, cardiac surgeon, LVAD coordinator, and social worker (in emergency situations at night, there may not be time for all evaluations to be undertaken). Because patients are often on a ventilator, or have mental status changes caused by cerebral hypoperfusion, this process generally relies on expeditiously deriving a comprehensive history from the family, discussions with referring physicians, a thorough clinical examination, and review of available diagnostic test data. The time factor does not generally permit performing additional diagnostic tests. In some instances, it may be impossible to derive a history and in that case, temporary mechanical support is preferred.

If no obvious contraindication to LVAD implantation or transplantation is identified, then, we proceed to operating room within 24 hours with intent to place a durable LVAD. For patients having drug boluses to maintain blood pressure, those deemed to be at risk of imminent cardiac arrest, and those referred with cardiac arrest who have return of spontaneous circulation, are taken emergently to surgery for implantation of a durable LVAD. If a patient is clearly not a transplant candidate, or unlikely to survive regardless of institution of mechanical support, then, medical therapy is continued and consideration given for hospice care. Unknown neurologic status, previous cardiac surgery, or recent administration of potent anticoagulants was not a contraindication to proceeding with durable LVAD.

This approach was applied only to patients with cardiogenic shock suspected to be secondary to AMI or ADHF. Patients with cardiogenic shock with other causes, such as postcardiotomy, myocarditis, and posttransplant allograft dysfunction, were treated on an individual basis, usually with short-term mechanical devices. Patients who have in-hospital cardiac arrest, without a previous diagnosis of cardiogenic shock caused by AMI or ADHF, were also excluded from this approach. Patients who have refractory cardiogenic shock caused by direct complication of percutaneous coronary intervention were also excluded.

Postoperative management was the same as for patients not in cardiogenic shock. Patients were formally evaluated for transplantation if they survived the immediate postoperative period. All patients were placed in a recovery protocol and maximized on neurohormonal blockade with 3monthly assessments for recovery using echocardiographic and right heart catheterization with device turndowns.

\section{Surgical Technique}

Patients were operated on either via median sternotomy or via our less invasive nonsternotomy approach. ${ }^{4,5}$ In all cases, the intention was univentricular support with an implantable LVAD, the exception being patients who had had cardiac arrest requiring cardiopulmonary resuscitation in the immediate preoperative period; in such cases, we electively placed a concurrent temporary RVAD (Centrimag Device 


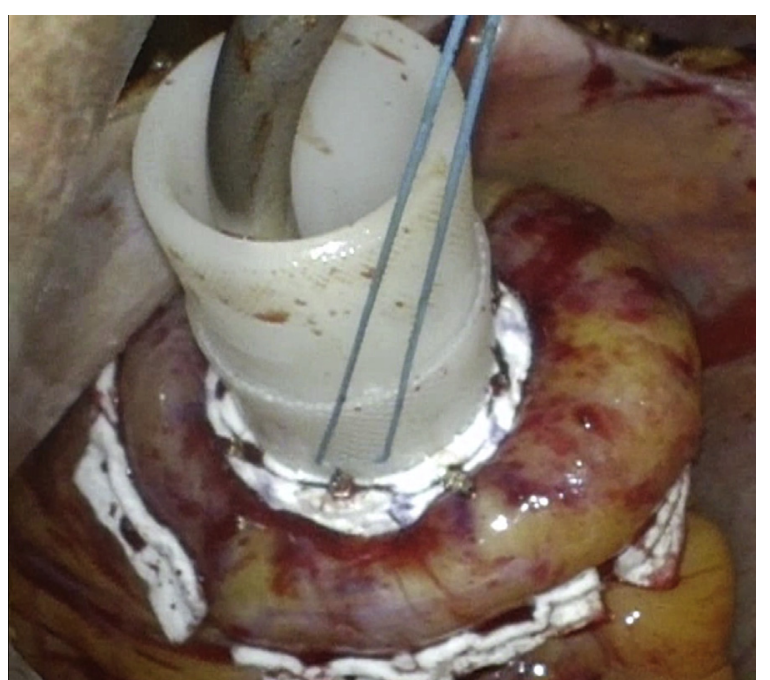

VIDEO 1. Case example: Emergency implantation of an implantable left ventricular assist device in cardiogenic shock. Video available at: http:// www.jtcvsonline.org/article/S0022-5223(17)32729-0/fulltext.

[St. Jude Medical, St. Paul, Minn]). Otherwise, RVADs were reserved for instances in which severe right ventricular failure after LVAD initiation could not be managed medically. Procedures were typically performed with cardiopulmonary bypass and without cardioplegic arrest. Concomitant procedures were performed as required. We have recently favored a nonsternotomy approach ${ }^{4}$ in critically ill patients with shock who do not require a concomitant procedure, particularly if coagulopathic bleeding is expected. The surgical technique is demonstrated in the Video 1.

Coagulopathy was corrected according to point-of-care testing and hemostasis obtained by prolonged surgical packing and additional suturing as required. The chest was closed after surgery only when acceptable hemostasis had been achieved (open chest management with delayed closure was not used in this cohort).

\section{Statistical Analysis}

Continuous variables were expressed as means and standard deviations if normally distributed or median and range for skewed distributions. Categorical variables were expressed as frequencies and proportions. Differences between groups were evaluated using the $\chi^{2}$ test or Fisher exact test for categorical variables as appropriate and independent samples Student $t$ test or the Mann-Whitney $U$ test for continuous variables depending on the distribution. The primary end point of survival was analyzed using the Kaplan-Meier method. Patients were censored at the time of heart transplantation, LVAD explantation, or last follow-up if neither transplantation nor explantation had occurred. The log-rank test was used to compare survival across groups. Survival estimates were derived from the life table and reported as percentage and standard errors. Cumulative incidence function for heart transplantation was constructed using competing risk analysis. All tests were 2 -tailed. A $P$ value $<.05$ was considered to be statistically significant. All statistical analyses were performed using SAS version 9.4 (SAS Institute, Cary, NC).

\section{RESULTS \\ Operative Data}

The predominant device used was Heartmate II (Abbott Laboratories, Lake Bluff, Ill) in 36 cases. Devices used in the remaining 7 patients were 1 Jarvik 2000 (Jarvik Heart,
NY), 1 Ventrassist (Ventracor, Chatswood, Australia), 2 Heartmate XVE (Thoratec, Pleasanton, Calif), and 3 HVAD (Heartware Inc, Framingham, Mass). One implant early in the series was performed off pump; cardiopulmonary bypass was used in all others, with a mean bypass time of 94 minutes. The mean operative time was $362 \mathrm{mi}-$ nutes. Nine operations $(21 \%)$ were performed less invasively with a nonsternotomy approach. Additional procedures were performed in 20 patients: tricuspid valve repair in 17 cases, mitral valve repair in 5 cases, aortic valve repair in 1 case, and coronary artery bypass grafting in 1 case (some patients had multiple procedures). Cardioplegic arrest was used in 1 case (this patient had aortic valve repair). Five patients (12\%) underwent concurrent RVAD insertion using centrally placed Centrimag device (Abbott Laboratories), 4 of whom had the RVAD successfully explanted at a later date via resternotomy (the other died on support). One patient underwent late implantation of RVAD on the tenth postoperative day; this was later successfully explanted.

\section{Early Outcomes}

Early perioperative outcomes are outlined in Table 2. Operative mortality was $14 \%$ ( 6 patients). Shock physiology could not be reversed in 4 patients who died as a result of progressive multiorgan failure on postoperative day $1,2,2$, and 5. One patient died of uncontrollable posterior nasopharyngeal bleed and sepsis on postoperative day 18, and the other one at 23 days postoperatively as a result of pneumonia, fungemia, and gastrointestinal bleeding. Three patients $(7 \%)$ required reoperation for postoperative bleeding, all from the ADHF subcohort. Twenty patients $(46 \%)$ were extubated within 48 hours of surgery. Overall,

TABLE 2. Operative variables and early outcomes

\begin{tabular}{lc}
\hline Sternal sparing incisions & $9(21)$ \\
Operative time (min) & $312(269-423)$ \\
\hline Cardiopulmonary bypass time (min) & $89(62-107)$ \\
\hline Reexploration for bleeding & $3(7)$ \\
\hline Right ventricular assist device & $6(14)$ \\
\hline Mechanical ventilation (d) & $3.5(1-9)$ \\
Mechanical ventilation $>1 \mathrm{wk}$ & $12(28)$ \\
Extubation within $48 \mathrm{~h}$ & $20(46)$ \\
Tracheostomy & $13(30)$ \\
\hline ICU stay (d) & $9(5-23)$ \\
\hline ICU stay more than $2 \mathrm{wk}$ & $16(37)$ \\
\hline Hospital stay (d) & $25(17-52)$ \\
\hline Hospital stay $>1$ mo & $18(42)$ \\
\hline Stroke & $2(4.7)$ \\
\hline Limb complications & $1(2.3)$ \\
\hline
\end{tabular}

Values are $\mathrm{n}(\%)$ or median (interquartile range) unless otherwise indicated. $I C U$, Intensive care unit. 


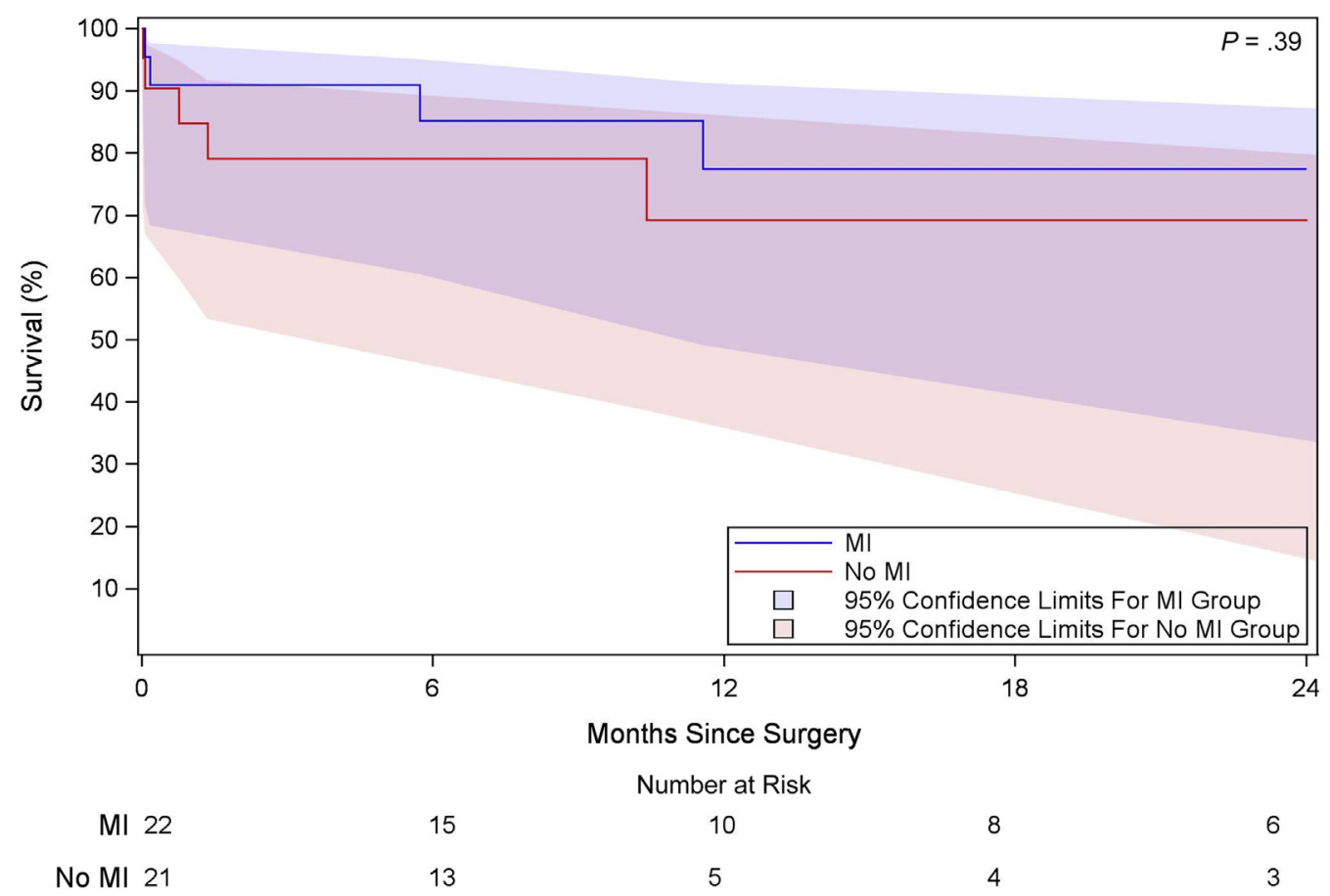

FIGURE 2. One-year survival stratified by myocardial infarction $(M I)$ versus non-MI.

the median cumulative time on the ventilator (including reintubations and prolonged weans) was 3.5 days. Median time in the intensive care unit (ICU) was 9 days (range, 1-85) and median hospital stay was 25 days (range, 1-142). Two patients $(5 \%)$ had postoperative stroke, one with complete neurologic recovery. In addition, a third patient who had prolonged cardiac arrest and resuscitation, and had unknown neurologic status before LVAD, woke up with occipital cerebral infarcts believed to be related to the cardiac arrest; he recovered and was subsequently transplanted.

\section{Midterm Outcomes}

Median follow-up was 10 months (with follow-up censored at time of heart transplantation). Actuarial survival censored for transplantation and device explantation for recovery was $82.7 \pm 6.0 \%$ at 6 months and $73.9 \pm 8.0 \%$ at 12 months. There was no significant difference in survival up to 2 years between AMI and ADHF groups (Figure 2). Using competing analysis, the cumulative incidence of transplantation was $10.3 \pm 5.0 \%$ at 6 months and $30.8 \pm 7.9 \%$ at 1 year.

Deaths within the first year (after operative period) included 1 patient at 42 days as a result of pneumonia, sepsis, gastrointestinal bleed, 1 at 6 months as a result of disconnection of the pump controller, 1 at 10 months as a result of sepsis, 1 at 11 months as a result of device thrombosis, and 1 at 11 months as a result of intracranial bleed. Three patients underwent device explantation. One patient underwent device explanation 8 months after implantation because of device thrombosis resulting from noncompliance in diagnosis of metastatic rectal carcinoma (see later discussion); he remained with advanced heart failure after LVAD explantation and died 2 months later. Another patient underwent device explanation at 97 days postoperatively after pump pocket infection; the left ventricular ejection fraction was $54 \%$ and the patient remained alive $>3$ years later. A third patient underwent device explantation for presumed recovery 13 months after implant but had return of advanced heart failure a few months later; she underwent placement of a second Heartmate II LVAD 8 months after the first was explanted and was subsequently successfully transplanted.

\section{Strategy-Specific Outcomes}

Of the 36 patients who had survived at least 3 months after hospital discharge at time of analysis, $30(83 \%)$ were listed for transplantation. Six patients were not listed for transplantation: 1 patient, aged 78 years at the time, was declined because of advanced age and remained alive $>4$ years later; another was diagnosed with interstitial lung disease and died 2.5 years later; another returned to alcohol abuse and died 11 months later from pump thrombosis; 1 was declined because of past lymphoma treatment and died of sepsis after 10 months while awaiting a malignancy-free interval; 1 died 6 months later of accidental device disconnection while evaluation for transplant was in progress; and the last patient was diagnosed with rectal carcinoma after a gastrointestinal bleed 3 weeks after LVAD placement and died 10 months later. 
Of 5 patients who had unknown neurologic status preoperatively, 3 had an intact neurologic examination postoperatively, 1 woke up with clinically evident occipital infarcts, and had near-total recovery, and 1 patient died on postoperative day 5 with progressive multiorgan failure; his neurologic status was never established.

\section{DISCUSSION}

We report good results with primary implantation of durable LVADs in refractory cardiogenic shock with more than $80 \%$ survival to hospital discharge. This finding is despite our cohort being very sick, including more than $50 \%$ on a ventilator, almost $20 \%$ with a history of cardiac arrest within hours of implant, and most patients having active hepatorenal dysfunction and coagulopathy. In addition, more than $40 \%$ had received potent antiplatelet drugs in the 24 hours before surgery. Our 1-year survival (74\%) was comparable to that for all-comer INTERMACS (Interagency Registry for Mechanical Assisted Circulatory Support) class 1 patients in the INTERMACS registry $(76 \%) .{ }^{6}$ Furthermore, despite undergoing emergent LVAD placement without formal transplant evaluation, most patients were listed for transplantation, so our strategy was reasonably effective in excluding inappropriate transplant candidates.

Our experience challenges the traditional arguments against the use of durable LVADs in cardiogenic shock, which are discussed in the following sections.

\section{Tolerance of Cardiopulmonary Bypass and Complex Surgical Operations by Patients in Refractory Cardiogenic Shock}

There has historically been a perception that patients in shock with multiorgan failure cannot tolerate cardiopulmonary bypass and long operations. ${ }^{7}$ Consequently, the mainstay of primary therapy for refractory cardiogenic shock has been short-term mechanical support devices. ${ }^{8-10}$ Our data show that it is possible to achieve success with durable LVADs in critically ill patients, despite a long and complex operation, and that these patients can tolerate cardiopulmonary bypass required for LVAD implantation. There is also a belief that the bleeding risk is prohibitive in patients in refractory shock, particularly those with hepatic dysfunction or those who have received potent antiplatelet or anticoagulant medications. With a modified surgical technique and protracted hemostasis, we were able to mitigate this bleeding risk; we did not leave any chest open, and less than $10 \%$ of patients were reexplored for bleeding. There were no reexplorations in the AMI group, despite most receiving potent antiplatelet therapy. Another concern with placing durable LVADs in acute cardiogenic shock is the friable apical myocardium often encountered in AMI. However, it has been shown that apical cannulation can be safely used in the setting of acute infarction. ${ }^{3,11,12}$ Twelve of 22 patients with AMI had histologically proven full-thickness or hemorrhagic acute infarction in the apical core; we did not experience any short-term or midterm adverse consequences of apical cannulation in these patients. Our experience, therefore, shows that emergency implantation of durable LVAD is technically feasible in refractory shock and can be safely and predictably applied.

\section{Resource Use}

Our patients spent an average of 3.5 days on a ventilator and 9 days in intensive care, which is a relatively short period for patients in refractory cardiogenic shock. ${ }^{13}$ Because of a low requirement for skilled monitoring and maintenance, we suspect that the use of durable LVADs allowed earlier discharge from ICU, and from hospital, which could otherwise not have been possible with traditional treatment approaches. One major observation was the infrequent need for reinterventions. Reinterventions result in additional operating room time, requirement for skilled staff, equipment and disposables used, prolongation of ICU stay, and additional burden from complications of reinterventions. In our series, only 7 patients $(17 \%)$ had repeat interventions, either for RVAD explants or reexploration for bleeding. For most patients ( $83 \%$ ), therefore, placement of a durable LVAD was a 1-stop therapy for cardiogenic shock, without any further surgical intervention. Alternative bridge-to-bridge strategies typically involve repeat interventions, such as for management of bleeding complications, left ventricular venting, limb perfusion, device exchanges, cannulation revision, exchange to short-term LVADs, and placement of durable LVADs, with potential for further reinterventions after any of these procedures. ${ }^{8}$

\section{Strategy-Specific Morbidity}

Morbidity specific to our strategy was low. We did not observe any early device-related complications (although after discharge, 2 patients died of device-related complications during follow-up). By avoiding peripheral cannulation with our primary LVAD approach, we observed nearelimination of limb complications, which are otherwise seen in as much as $17 \%$ of patients being treated with conventional strategies ${ }^{14}$ (1 patient in our current series had limb ischemia related to a preoperative intra-aortic balloon pump). Although limb complications can also occur in patients having durable LVADs, the incidence is substantially lower compared with patients who receive either temporary devices or ECMO. ${ }^{15}$

\section{Implantation of Durable LVAD Without Formal Evaluation}

A major drawback of our approach is that we placed durable LVADs without a formal patient evaluation, sometimes within hours of first contact of the patient with the 
LVAD team. This strategy risks the possibility of discovering, after the surgery, that a durable LVAD has been inadvertently placed in a patient who has contraindications to LVAD therapy, or who may lack adequate social structure necessary to achieve an effective result. A thorough history and rapid medical and social screening are therefore critical before emergency implantation of durable LVADs. If there is any doubt, a temporary device should be used while candidacy is being clarified. Using this strategy, we had 1 patient implanted with a durable LVAD who subsequently turned out to be a poor social candidate (an alcoholic who returned to drinking and became noncompliant with therapy) and another who turned out to have a medical contraindication (a patient with undiagnosed rectal carcinoma). Most patients treated with our strategy were listed for transplantation, validating the effectiveness of our rapid screening strategy.

\section{Implantation of a Durable LVAD When There Is Possibility of Early Cardiac Recovery}

Some would argue that it is excessive to place a durable LVAD in patients with primary shock because the myocardium could recover. However, we did not observe clinically relevant cardiac recovery in most patients, despite systematic screening for recovery. Although our experience contrasts with those of others who have achieved higher level of weaning to recovery with short-term devices placed for refractory cardiogenic shock, ${ }^{16}$ even with a high rate of successful weaning of ECMO, many of the weaned patients do not survive the first year. ${ }^{13,16,17}$ This finding suggests that sustained recovery may not be truly achievable in many patients with refractory shock; one of the patients in whom we explanted the LVAD for presumed recovery had a recurrence of advanced heart failure several months later requiring reimplantation of an LVAD and subsequent transplantation.

\section{Limitations}

This is a small single-center retrospective study and has all the inherent limitations associated with this study design. In addition, the patient population reflects a selected study group of patients who met our criteria (Figure 1) so cannot be generalized to every patient with cardiogenic shock. Because most surviving patients were censored because of transplantation, few patients were available for midterm survival analysis. We have not captured long-term INTERMACS device-related outcomes; our focus was on outcomes of cardiogenic shock, primarily survival, and recovery, as opposed to devicerelated complications (which is not applicable to general cardiogenic shock cohorts). Because of the retrospective design, we cannot provide the denominator of patients treated in our institution for refractory cardiogenic shock, including those treated with medical therapy and palliative care. The most notable limitation is the lack of a control group, which prevents determination of the effectiveness of our strategy and direct comparison with alternative approaches; short-term devices, ECMO, medical therapy, and hospice care are complementary to durable LVADs in our approach (Central Figure) and are applied in patients if futility is suspected either in the short-term or long-term or if candidacy for advanced surgical therapies cannot be easily confirmed. Given strict reimbursement and regulatory rules that govern LVAD implantation in many localities, ours may not be a practical approach if listing for transplantation is required before LVAD implantation.

The concept of primary LVAD implantation is not directly applicable to all centers. Undertaking emergency implantation of durable LVADs requires the presence of appropriate infrastructure and a surgical team agreeable and able to perform such surgery round the clock. Furthermore, because surgeons have different styles and philosophies regarding operative management, our approach may not be applicable to surgeons who have a different operative approach from ours. For example, surgeons have to be adaptable to suturing device inflow to recently infarcted myocardium, operating on patients shortly after administration of potent antiplatelet drugs, performing redo procedures emergently without preoperative imaging, using minimal access approaches in appropriate cases, and be committed to avoiding RVADs in most of these patients. We also believe patience and commitment to complete hemostasis before leaving the operating room are critical to success with this technique. Our operations took an average of 5 hours, and a quarter of procedures took longer than 7 hours; prolonged periods of hemostasis were often necessary and contributed to this long operative time. Strategies that require leaving the chest open for delayed closure after LVAD implantation ${ }^{18}$ may not be compatible with the emergent LVAD scenario. Avoidance of RVADs in critical cardiogenic shock requires patient weaning from bypass and prolonged periods of observation under inotropic management; the alternative strategy of liberal RVAD implantation is unlikely to achieve similar results to ours because of need for reinterventions to remove RVAD, likely more bleeding complications, and longer ICU stay.

\section{CONCLUSIONS}

We have systematically performed primary implantation of durable LVADs for refractory cardiogenic shock caused by AMI or ADHF as a one-stop strategy with excellent early and midterm outcomes. Our experience provides a proof of concept that durable LVADs can be effectively applied for refractory cardiogenic shock. Further studies are required to ascertain whether these results can be replicated in other centers and whether 
a strategy of primary LVAD implantation results in benefits compared with traditional bridge-to-bridge approaches.

\section{Webcast}

You can watch a Webcast of this AATS meeting presentation by going to: http://webcast.aats.org/2016/Video/ Tuesday/05-17-16_Ballroom_I_0803_Pawale-800.mp4.

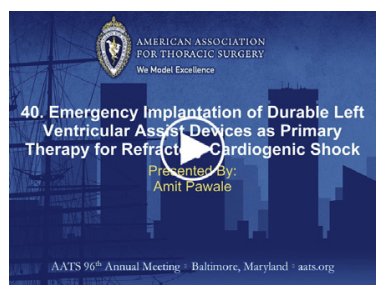

\section{Conflict of Interest Statement}

Yosef Schwartz was a 2015 AATS summer intern. Dr Adams receives royalties from Edwards Lifesciences, Medtronic (to Icahn School of Medicine at Mount Sinai), and National Co-PI with Medtronic on transcatheter valve trials. Dr Pinney is a consultant for Abbott Laboratories. All other authors have nothing to disclose with regard to commercial support.

\section{References}

1. Aggarwal S, Slaughter MS. Acute myocardial infarction complicated by cardiogenic shock: role of mechanical circulatory support. Expert Rev Cardiovasc Ther. 2008;6:1223-35.

2. Feldman D, Pamboukian SV, Teuteberg JJ, Birks E, Lietz K, Moore SA, et al. The 2013 International Society for Heart and Lung Transplantation Guidelines for mechanical circulatory support: executive summary. J Heart Lung Transplant. 2013;32:157-87.

3. Pawale A, Pinney S, Ashley K, Flynn R, Milla F, Anyanwu AC. Implantable left ventricular assist devices as initial therapy for refractory postmyocardial infarction cardiogenic shock. Eur J Cardiothorac Surg. 2013;44:213-6.

4. Anyanwu AC. Technique for less invasive implantation of Heartmate II left ventricular assist device without median sternotomy. Semin Thorac Cardiovasc Surg. 2011;23:241-4.

5. Anyanwu AC, Itagaki S, Pinney S, Adams DH. Initial experience with routine less invasive implantation of HeartMate II left ventricular assist device without median sternotomy. Eur J Cardiothorac Surg. 2014;46:985-90.

6. Kirklin JK, Naftel DC, Pagani FD, Kormos RL, Stevenson LW, Blume ED, et al. Seventh INTERMACS annual report: 15,000 patients and counting. J Heart Lung Transplant. 2015;34:1495-504.

7. Frazier OH, Gregoric ID, Cohn WE. Initial experience with non-thoracic, extraperitoneal, off-pump insertion of the Jarvik 2000 Heart in patients with previous median sternotomy. J Heart Lung Transplant. 2006;25:499-503.

8. Takayama H, Soni L, Kalesan B, Truby LK, Ota T, Cedola S, et al. Bridge-to-decision therapy with a continuous-flow external ventricular assist device in refractory cardiogenic shock of various causes. Circ Heart Fail. 2014;7:799-806.

9. Negi SI, Sokolovic M, Koifman E, Kiramijyan S, Torguson R, Lindsay J, et al. Contemporary use of veno-arterial extracorporeal membrane oxygenation for refractory cardiogenic shock in acute coronary syndrome. J Invasive Cardiol. 2016;28:52-7.

10. Rihal CS, Naidu SS, Givertz MM, Szeto WY, Burke JA, Kapur NK, et al. 2015 SCAI/ACC/HFSA/STS Clinical Expert Consensus Statement on the Use of Percutaneous Mechanical Circulatory Support Devices in Cardiovascular Care: Endorsed by the American Heart Association, the Cardiological Society of India, and Sociedad Latino Americana de Cardiologia Intervencionista; Affirmation of Value by the Canadian Association of Interventional Cardiology-Association Canadienne de Cardiologie d'intervention. J Am Coll Cardiol. 2015;65:2140-1.
11. Park SJ, Nguyen DQ, Bank AJ, Ormaza S, Bolman RM III. Left ventricular assist device bridge therapy for acute myocardial infarction. Ann Thorac Surg. 2000;69: 1146-51.

12. Leshnower BG, Gleason TG, O'Hara ML, Pochettino A, Woo YJ, Morris RJ, et al. Safety and efficacy of left ventricular assist device support in postmyocardial infarction cardiogenic shock. Ann Thorac Surg. 2006;81: $1365-70$.

13. Tarzia V, Bortolussi G, Bianco R, Buratto E, Bejko J, Carrozzini M, et al. Extracorporeal life support in cardiogenic shock: impact of acute versus chronic etiology on outcome. J Thorac Cardiovasc Surg. 2015;150:333-40.

14. Cheng R, Hachamovitch R, Kittleson M, Patel J, Arabia F, Moriguchi J, et al. Complications of extracorporeal membrane oxygenation for treatment of cardiogenic shock and cardiac arrest: a meta-analysis of 1,866 adult patients. Ann Thorac Surg. 2014;97:610-6.

15. Ohman JW, Vemuri C, Prasad S, Silvestry SC, Jim J, Geraghty PJ. The effect of extremity vascular complications on the outcomes of cardiac support device recipients. J Vasc Surg. 2014;59:1622-7.

16. Xie A, Phan K, Tsai YC, Yan TD, Forrest P. Venoarterial extracorporeal membrane oxygenation for cardiogenic shock and cardiac arrest: a meta-analysis. $J$ Cardiothorac Vasc Anesth. 2015;29:637-45.

17. Bermudez CA, Rocha RV, Toyoda Y, Zaldonis D, Sappington PL, Mulukutla S, et al. Extracorporeal membrane oxygenation for advanced refractory shock in acute and chronic cardiomyopathy. Ann Thorac Surg. 2011;92:2125-31.

18. Quader M, LaPar DJ, Wolfe L, Ailawadi G, Rich J, Speir A, et al. Delayed sternal closure after continuous flow left ventricle assist device implantation: analysis of risk factors and impact on outcomes and costs. ASAIO J. 2016;62: 432-7.

Key Words: cardiogenic shock, heart failure, heart transplantation, left ventricular assist device, myocardial infarction

\section{Discussion}

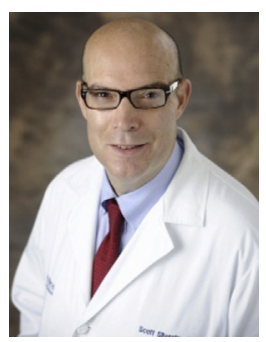

Dr S. Silvestry (Orlando, Fla). Thank you for the opportunity to discuss this paper and presenting the paper in advance. The authors reported their experience with acute implantation of durable LVADs in patients with refractory cardiogenic shock with excellent outcomes. This study highlights several important observations that remain true despite practice trends in the opposite direction and that potentially, depending on your location to Central Park, will depend on whether or not you get a durable LVAD or a temporary support.

But durable LVADs are excellent acute circulatory support devices. Supporting the patient by increasing perfusion at the right time improves outcomes, decreases length of stay, and decreases cost of care when done correctly. And acutely ill patients have significant survival benefits with LVAD therapy when aggressively treated. These are shown in your paper and remain true despite trends in the opposite direction.

I have several questions. One, shock is a double-edged sword. How much organ dysfunction is too much when you assess the patient? Do you use risk scores or organ dysfunction scores to gauge who is too sick to go directly to an LVAD? 


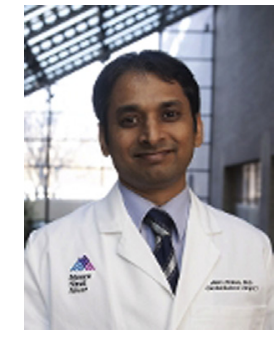

Dr A. Pawale (New York, NY). We did not use any clinical score and it was clinical judgment.

Dr Silvestry. Although the cohort is labeled refractory cardiogenic shock, the timing of LVAD implants in the cohort is variable for both subgroups with acute MI and acute decompensated heart failure. What proportion of the entire cohort was implanted within days to 1 week of shock presentation, because there is a range as much as 23 days out from that, and acute shock is different than chronic shock.

Dr Pawale. In the acute myocardial infarction group, the median time from infarct to implant was about 3 days, and in refractory cardiogenic shock due to acute decompensated heart failure, retrospectively it was hard to determine when the onset of shock was, but once the patient was referred with refractory shock, then he was implanted within 24 hours.

Dr Silvestry. Your RVAD use was $14 \%$ in this acute setting. In our INTERMACS 1 cohort it was 7\%, which we thought was high. Do you think that there is an opportunity to benefit from these patients using nondurable LVADs acutely and perhaps resuscitating them further? Can you identify these patients or make any comments on these patients in particular?

Dr Pawale. This group of patients is in refractory cardiogenic shock, like INTERMACS 0 . So it is not the exact comparison with the INTERMACS 1 group. However, our series has a $12 \%$ RVAD implantation, which is $12 \%$ biventricular support as compared to the other alternative strategy of ECMO, which is almost $100 \%$ biventricular support. And as we saw today with ECMO support, RVAD use was $27 \%$ and in our group it is $14 \%$. So we think it is less RVAD use in our group.

Dr Silvestry. You are aware there is no real INTERMACS 0.

Four patients in your group had persistent shock and died within 5 days with LVAD therapy. Does this again give you an opportunity to identify which patients may have benefited from ECMO, et cetera?

Dr Pawale. We believe that there is a small group of patients in whom their end-organ dysfunction has reached to an irreversible stage. So in spite of restoring full cardiac output irrespective of the device used, they progress into multiorgan failure in spite of reversal of shock. So maybe they had delayed institution of mechanical support.

Dr Silvestry. Eighty percent of your patients ultimately who survived to discharge were listed for transplant. So your evaluation was very much spot on. How long did it take and how many patients fell out during that rapid evaluation? I am pretty good with insurance companies, but it still takes 2 days to get approval for implant.

Dr Pawale. It took probably 3 to 4 hours for a rapid evaluation with the social worker, heart failure cardiologist and surgeon, and most of the times financial barriers were met eventually.

Dr Silvestry. And then the last question is, despite the fact that LVADs are exceptional LV decompression as we debate ECMO and whether decompression or not, there was only 1 patient that had a recovery with acute MI. This is something that has been seen in many series, in our series and others. Can you offer an explanation? Do you think perhaps that continuous-flow LVADs are bad for recovery, are not a good recovery mode without training?

You had excellent results. Thank you for the opportunity to discuss your paper.

Dr Pawale. Thank you. It is possible that continuousflow devices have less weaning potential than pulsatile devices. However, this group of patients particularly had large myocardial infarction, many times diffuse coronary disease, had acute and chronic decompensation of heart failure. So these are usually not a weanable group of patients. There are no predictable definitive algorithms. So even in the ECMO group of patients for this particular group, the weaning potential is very small, and after weaning, patients can have recurrent or persistent heart failure and mortality. So I think it is still acceptable. As compared to having $50 \%$ survival at the end of 1 year with a temporary bridge-to-bridge approach, our survival is probably better.

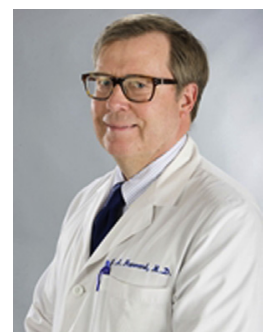

Dr J. Hammon (Hartford, Conn). Unbelievable results. You are to be congratulated. It looked like to me that your average OR time was about 6 hours, although your pump runs weren't all that long, which leads me to believe you spent an awful lot of time in the operating room fine tuning the patients before you came out.

How many patients came out of the OR, for example, with the chest open or were you able to close it primarily every time?

Dr Pawale. None of the patients had open chest coming out of the OR. We took prolonged hemostasis and laborious efforts to have packing, repacking, and correction of coagulopathy with point-of-care testing. We also had a higher threshold for RVAD support, and we tried to optimize the $\mathrm{RV}$ intraoperatively. So that is why our operating time is longer.

Dr Hammon. Amazing patients. We have often been concerned in the setting of an acute MI that the left ventricular cavity might be small and therefore be a point of obstruction for inflow. Did you have any concerns with that? Did you take any special measures to align the cannula?

Dr Pawale. No. Even if the patient had an acute MI and the LV cavity was not enlarged, we did not 
have any problem with cannula position, unlike the restrictive cardiomyopathy patients. So we did not have any problem.

Actually out of our acute MI group, 12 patients had transmural infarct of the apex on the pathological examination postoperatively, but with meticulous technique we did not have any issues of the apex falling apart.

Dr Hammon. So no acute MI was ever turned down because of size of the left ventricle?

\section{Dr Pawale. No.}

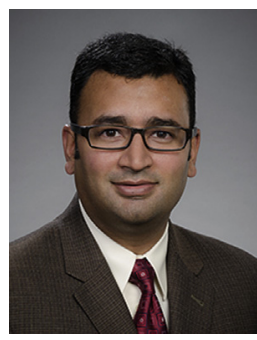

Dr N. Mokadam (Seattle, Wash). How do you deal with payment? The patients you are describing were not listed for transplant presumably and did not meet DT criteria. You run the risk of running into financial troubles with that. Can you comment on that?
Dr Pawale. We did not have any major issues with reimbursement, and in terms of cost effectiveness, we believe that with a durable LVAD, with an implantable device, the cost may be lower with less recurrent intervention, less ECMO-related complications and less hospital stay. But we did not have major issues with reimbursement.

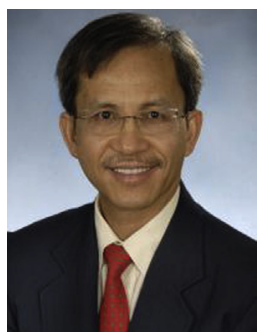

Dr S. Pham (Baltimore, Md). Cardiogenic shock patients can develop acute renal failure after these major operations. What is your incidence of dialysis during and after hospitalization in these patients?

Dr Pawale. We did not have any long-term dialysis requirement in any of these patients. When we screen the patients, we evaluate the patients if they have acute renal failure rather than a long-term renal process. So we did not have any long-term dialysis. Some patients required temporary support. 